\title{
A Literature Review of Localized Mediastinal Mesothelioma and a Rare Case Report
}

\author{
Qiang Meng Xiao', Meng Zhang ${ }^{1}$, Fengjun du$^{2}$, Hui Peng ${ }^{3}$, Jun Chen ${ }^{1}$, Hailong \\ Liu $^{1}$
}

${ }^{1}$ Radiology Department, Zhuhai Hospital of Guangdong Hospital of Traditional Chinese Medicine, China. ${ }^{2}$ Cardio-Thoracic Surgery, Zhuhai Hospital of Guangdong Hospital of Traditional Chinese Medicine, China. ${ }^{3}$ Pathology Department, Zhuhai Hospital of Guangdong Hospital of Traditional Chinese Medicine, China.

\begin{abstract}
Background: Primary localized mediastinal mesothelioma is a rare tumour which accounts for about $0.7 \%$ of all mesotheliomas. The mesothelial cells lining of the pericardium are suggested as the most probable cells of origin. Mediastinal pleural mesothelioma are distinctly uncommon, which has only been reported in a single case. Case presentation: We report a case of a 57 years old man with primary mediastinal pleural mesothelioma involving middle and posterior upper mediastinum, one months following surgery, the patient is alive with good performance status. Chest plain scan and contrast-enhanced computer tomography (CT) scan showed a $43 \mathrm{~mm} \times 36$ $\mathrm{mm} \times 36 \mathrm{~mm}$ tumor, with a CT value of about $41.5 \mathrm{HU}$, an arterial phase CT value of about $59.8 \mathrm{HU}$ and a venous phase CT value of about $77.5 \mathrm{HU}$ during the contrast-contrast-enhanced scan. Patients underwent thoracotomy. The pathological diagnosis was derived from malignant pleural mesothelioma (MPM) growing from the chest wall into the mediastinum. Conclusions: Imaging manifestations of tumors have rarely been described in the literature. A short review of literature on MPM is given and summarize the imaging characteristics.
\end{abstract}

Keywords: Localized malignant mesothelioma- mediastinal tumor- pericardial malignant mesothelioma- rare tumour

Asian Pac J Cancer Care, 6 (1), 131-135

\section{Introduction}

Mediastinal mesothelioma, mainly malignant pleural mesothelioma (MPM), is rare among primary cardiac and pericardial tumors, and its incidence ranges from $0.0017 \%$ to $0.33 \%$ in case reports or collected autopsies [1]. Pericardial MPM is a rare and fatal pericardial mesothelial surface disease, accounting for $0.7 \%$ of all mesothelioma cases, with an incidence of 1 in 40 million; in 500,000 autopsies, it has an incidence of $0.0022 \%$ [2]. MPM may be present in the pleura $(70-75 \%)$, rarely in the peritoneum $(20-25 \%)$, pericardium $(4 \%)$, or rarely elsewhere, and the incidence of localized mediastinal mesothelioma is even lower, which has only been reported in a single case [3-4]. In this report, we described the clinical presentation, imaging findings, intraoperative findings, histopathology, and immunohistochemistry of an incidentally discovered localized mesothelioma of the
Submission Date: 12/17/2020Ａcceptance Date: 02/11/2021

\footnotetext{
Corresponding Author:

Dr. Hailong Liu

Radiology Department, Zhuhai Hospital of Guangdong Hospital of Traditional Chinese Medicine, China.

Email: yueting1452@163.com
}

upper mediastinum and reviewed the relevant literature. According to the literature I reviewed, this paper is the first to summarize the imaging characteristics of all localized mediastinal mesothelioma cases.

\begin{abstract}
Case report
A 57-year-old male presented with back pain without obvious inducement 9 months ago. At that time, the patient had no chest distress pain, sweating, fatigue, nausea, vomiting, abdominal pain, abdominal distension, diarrhea, etc., which could be relieved spontaneously. Later, the patient gradually developed chest distress pain, located behind the sternum, without limb cold sweating, nausea, vomiting, acid reflux, heartburn or other discomforts during the pain. About half an hour after onset, the pain could be relieved spontaneously. The patient
\end{abstract}


Table 1. Diagnosis and Treatment Process

\begin{tabular}{|c|c|c|c|c|}
\hline Year month & $2019-8$ & $2020-1$ & $2020-05$ & $2020-7$ \\
\hline $\begin{array}{l}\text { Diagnosis and } \\
\text { treatment process }\end{array}$ & Back pain & $\begin{array}{l}\text { Coronary CTA incidentally } \\
\text { revealed a tumor }\end{array}$ & Thoracotomy surgery & $\begin{array}{l}\text { No radiotherapy and chemotherapy, } \\
\text { The follow-up visit recovered well }\end{array}$ \\
\hline
\end{tabular}

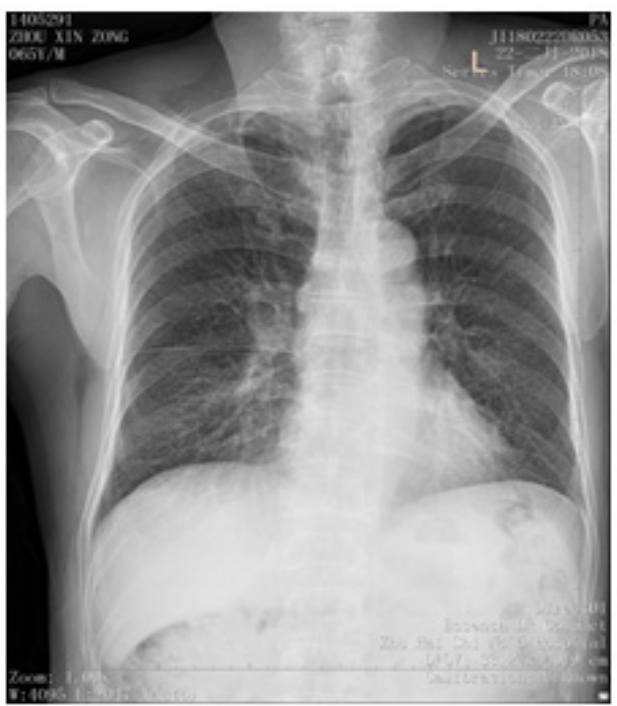

Figure 1. Anteroposterior Chest Radiograph Showed no Abnormality

had no obvious history of asbestos exposure. Physical examination revealed no abnormality. Coronary CTA incidentally revealed a tumor in the middle and posterior upper mediastinum (Table 1).

Imaging findings: An anterior chest radiograph done 8 months revealed no abnormalities (Figure 1). The two-dimensional B-ultrasound image showed a mild hypoechoic mass in the upper mediastinum with clear boundaries, oval shape, uneven internal echo and no change in tail echo. Color Doppler flow imaging showed no obvious blood flow signal (Figure 2). Plain scan and contrast-enhanced CT scan showed a $43 \mathrm{~mm}$ $\times 36 \mathrm{~mm} \times 36 \mathrm{~mm}$ tumor in the middle and posterior superior mediastinum, with a CT value of about $41.5 \mathrm{HU}$, an arterial phase CT value of about $59.8 \mathrm{HU}$ and a venous phase CT value of about $77.5 \mathrm{HU}$ during the contrastcontrast-enhanced scan, with heterogeneous moderate enhancement; the tumor was adjacent to the ascending aorta, trachea, superior vena cava and right pulmonary artery. It was closely connected to the left chest wall, abutting the aortic wall, with a rough margin and indistinct demarcation from the surroundings, and no pleural or pericardial effusion was seen (Figuer 3).

Multidisciplinary consultation in our hospital, suspected mediastinal malignancy, and detailed systemic examination revealed no other metastatic or primary lesion, and we performed a thoracotomy. Intraoperative findings: There was a mass above the aortic arch of the posterosuperior mediastinum and in the left rear of the esophagus (about T3 - 5 level), which abutted the esophagus and aortic arch and was tightly attached to the left chest wall, and the size of the mass was about 30 $\mathrm{mm} \times 25 \mathrm{~mm} \times 60 \mathrm{~mm}$, which was hard and had poor range of motion.

Most of the tissues were cystic wall-like, with fibrous collagenization of the capsule wall accompanied by more lymphocyte infiltration; atypical cells showed papillary, patchy, pseudo glandular, and infiltrative growth in the focal part near the surface area; tumor cells were polymorphic, mainly shuttle-shaped and oval, with abundant red staining of the cytoplasm, irregular karyotype, and visible mitoses (Figure 4). Immunohistochemical results: TTF-1 (-), Napsin A (-), CK7 (partial +), P40 (-), P63 (-), CK5/6 (+), Syn (-), CgA (-), CD56 (-), Ki67 (50\% +), MC (+), CK (+), Desmin (-), EMA (+), P53 (70\% +), E-cad (cytoplasmic $+)$, vimentin (+), CD5 (-), TdT (-), CD117 (-), CEA (-), PAX8 (-), CD31 (-), CD34 (-), Calretinin (good delivery, issued results -), WT1 (good delivery, issued results -). The pathological diagnosis in our hospital was derived from MPM growing from the chest wall into the mediastinum.

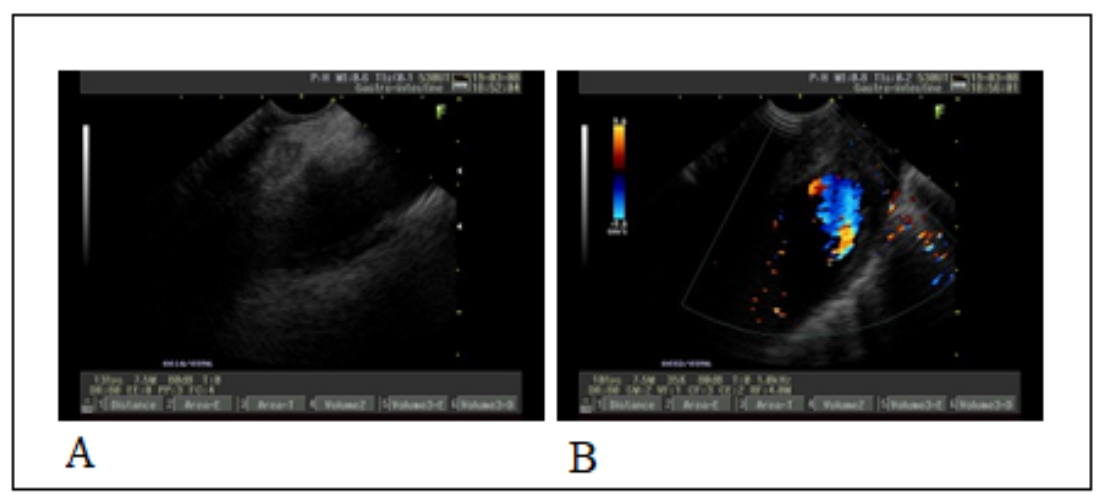

Figure 2. Transesophageal Ultrasonography, A, Slightly Hypoechoic Mass with Clear Boundary, Oval Shape, about $12 \mathrm{~mm} \times 6 \mathrm{~mm}$ in Size Can be Seen in the Upper Mediastinum Area on the Two-dimensional B-ultrasound Image. The internal echo is not uniform, and there is no change in the rear echo. B, Color doppler flow imaging showed no obvious blood flow signal 
Table 2. The Imaging Characteristics and Reported Cases of MPM

\begin{tabular}{|c|c|c|c|c|c|}
\hline Case & Age/Sex & $\begin{array}{l}\text { Predisposing } \\
\text { factors of asbestos } \\
\text { exposure }\end{array}$ & $\begin{array}{l}\text { Tumor } \\
\text { size } \\
(\mathrm{CM})\end{array}$ & CT findings & MRI findings \\
\hline $1[14]$ & $27 /$ female & NO & 4 & NO & $\mathrm{NO}$ \\
\hline $2[15]$ & $50 /$ male & $\mathrm{NO}$ & 10 & $\mathrm{NO}$ & NO \\
\hline $3[16]$ & 44/female & $\mathrm{NO}$ & 11 & NO & $\begin{array}{l}\text { Mass located above the heart, and } \\
\text { below the aortic arch and pulmonary } \\
\text { artery on T1WI }\end{array}$ \\
\hline $4[17]$ & $52 / \mathrm{male}$ & NO & 7 & $\begin{array}{l}\text { A contrast-enhanced CT scan showed a } 43 \times 36 \mathrm{~mm} \\
\text { mediastinal tumor adjacent to the ascending aorta, trachea, } \\
\text { superior vena cava, and right pulmonary artery. The mass } \\
\text { was well encapsulated. Inhomogeneous and faint } \\
\text { enhancement was observed in the arterial and delayed } \\
\text { phases. No pleural or pericardial effusion was seen. }\end{array}$ & \\
\hline $5[18]$ & $59 /$ female & NO & 6 & $\begin{array}{l}\text { Pericardial effusion, enhanced CT image findings were } \\
\text { basically the same as those in muscles. }\end{array}$ & $\begin{array}{l}\text { Pericardial effusion, enhanced MRI } \\
\text { image findings were basically the } \\
\text { same as those in muscles. }\end{array}$ \\
\hline 6 [19] & $68 /$ male & NO & $\begin{array}{l}3.5 \\
\text { (surgery) }\end{array}$ & $\begin{array}{l}\text { CT scan showed a } 36 \times 22 \mathrm{~mm} \text { pericardial tumor with } \\
\text { density basically the same as muscle, pericardial } \\
\text { effusion, moderate enhancement after contrast-enhanced } \\
\text { scan, which was higher than muscle and lower than the } \\
\text { aorta. }\end{array}$ & No \\
\hline 7 [7] & $74 /$ male & YES & 6 & $\begin{array}{l}\text { Contrast-enhanced chest CT showed a right anterior } \\
\text { mediastinal mass measuring } 60 \mathrm{~mm} * 50 \mathrm{~mm} * 40 \mathrm{~m} \text { in } \\
\text { size, which was mainly of the cystic component. Smooth } \\
\text { and firm cyst wall, with a uniform thickness; the mass also } \\
\text { had soft tissue components closely related to the ascending } \\
\text { aorta, there was no plane of separation between the tumor } \\
\text { and the aorta, and the density was essentially the same as } \\
\text { that of the muscle after } 3 \text { months showed recurrent mass at } \\
\text { the same site. This mass was mainly of soft tissue } \\
\text { attenuation without a remarkable cystic component. mild } \\
\text { enhancement after contrast-enhanced scan. }\end{array}$ & $\begin{array}{l}\text { The recurrent mass was hyperintense } \\
\text { on both } \mathrm{T} 1 \text { and } \mathrm{T} 2 \text {, Diffuse and } \\
\text { homogeneous contrast enhancement } \\
\text { of the mass was noted }\end{array}$ \\
\hline 8 [12] & 76/female & NO & 3.6 & NO & NO \\
\hline 9 & $57 /$ male & NO & 4.3 & $\begin{array}{l}\text { CT scan showed a } 43 \mathrm{~mm} \times 36 \mathrm{~mm} \times 36 \mathrm{~mm} \text { tumor in the } \\
\text { middle and posterior superior mediastinum, with } \\
\text { remarkable manifestations in the middle mediastinum, } \\
\text { adjacent to the ascending aorta, trachea, superior vena cava } \\
\text { and right pulmonary artery, with soft tissue density and } \\
\text { moderate progressive homogeneous enhancement after the } \\
\text { contrast-enhanced scan. }\end{array}$ & NO \\
\hline
\end{tabular}

\section{Follow-up and Outcomes}

There has been significant progress in the treatment of malignant mesothelioma, although it remains extremely difficult to treat.

\section{Discussion}

MPM is a rare malignant tumor that is invasive and generally produces mesothelial cells such as pericardium, pleura, and peritoneum. Thoracic MPM is the most common, accounting for approximately $70 \%-80 \%$ in terms of the total incidence of MPM, followed by malignant peritoneal mesothelioma [5]. MPM is characterized by a long latency period, a high degree of malignancy, and a poor prognosis. The pathogenesis of the disease is still unclear. At present, asbestos exposure is considered to be an important predisposing factor, and tuberculosis, radiation, thorotrast, etc. are also one of the predisposing factors [6-8]. Now more and more reported patients do not have a confirmed history of asbestos exposure. The main clinical symptoms are cough, chest pain, chest tightness, dyspnea, etc., and the clinical manifestations have no specificity, which brings great difficulties to its diagnosis and subsequent treatment.

Primary MPM of the pericardium accounts for less than $5 \%$ of diagnosed mesotheliomas [9]. It usually presents as multiple pericardial nodules, with or without a dominant mass, or a diffuse tumor, encasing at least a portion of the heart. There are three types of reports: extrapericardial disease $(10 \%)$; localized pericardial disease $(15 \%)$; diffuse nodular tumors, which can invade the heart (75\%) [10-11]. Localized (solitary) pericardial mesothelioma is considered a rare variant of MPM. In 2002, J.Fernando et al. [12] reviewed only 4 cases in the literature, and S.Cao et al. [13] reported 8 cases in 2017, an increase of 4 cases compared with before. Marchevsky et al. [14] reviewed 101 cases of localized mesothelioma reported in the English literature before July 2019, which did not show an increase in the number of cases of mediastinal mesothelioma. We reviewed the English literature before May 2020, and found only 8 cases of localized (solitary) mesothelioma, with the reported cases shown in Table 2. Together with our case, there were a total of 9 cases, our case was mediastinal pleural mesothelioma, and the rest were pericardial mesothelioma. Among the 9 cases, there was only one patient with a history of predisposing 


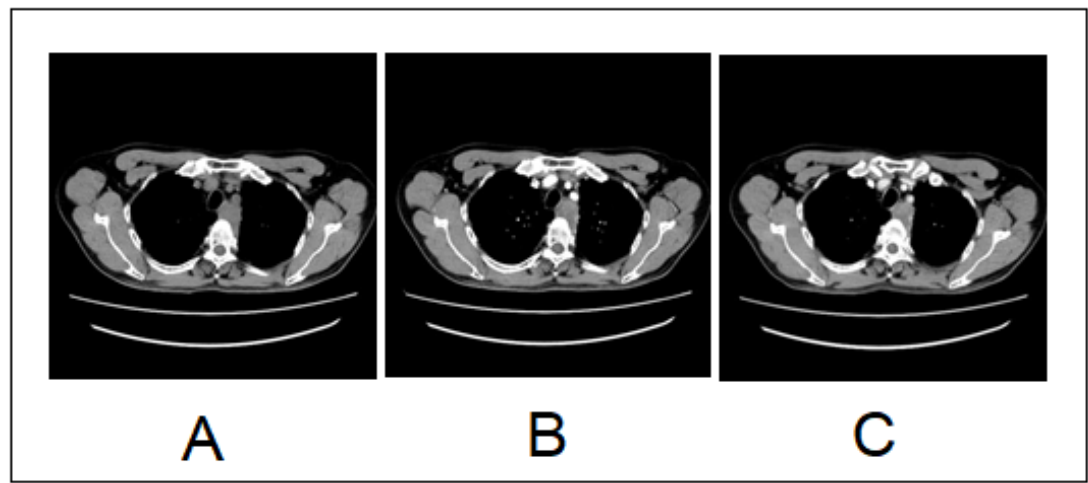

Figure 3. A, is Plain CT Scan; B, is Contrast-enhanced CT Scan During the Arterial Phase; C, is Contrast-enhanced CT Scan During the Venous Phase, the Mass is Located in the Upper, Middle and Posterior mediastinum, Abutting the Pleura, with Progressive Moderate Enhancement after a Contrast-enhanced Scan.

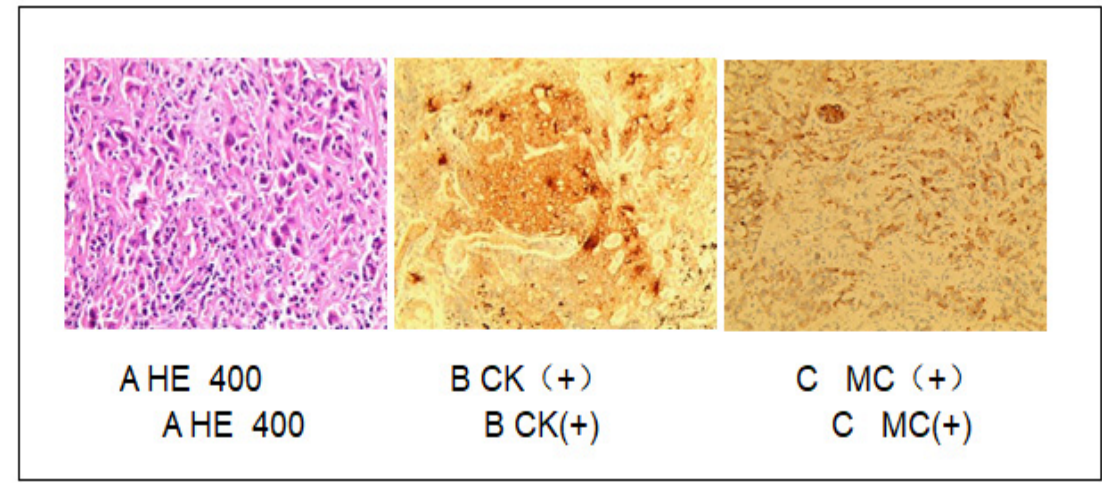

Figure 4. A, Tumor Cells were Polymorphous, Mainly Shuttle and Oval, with Abundant Red Staining of the Cytoplasm, Irregular Karyotype, and Visible Mitoses; B, CK Immunochemical Staining was Positive; C, MK Immunochemical Staining was Positive.

factors, all of which were malignant tumors, involving 4 females and 5 males. We analyzed 3 patients with only CT scan data and 1 patient with only MRI contrastcontrast-enhanced scan data, and 1 patient with CT contrast-enhanced scan data and MRI contrast-enhanced scan data. In 4 articles, there were only CT images or MRI images, The CT image findings were not described in the article. Therefore, two senior chest attending physicians in our hospital analyzed the CT image or MRI image in the literature and our case, respectively, and obtained the image findings after discussion following listening to different views. Results showed that the image findings showed 4 cases of localized soft tissue mass, and 1 case of cystic solid lesion (cystic dominantly), after 3 months showed recurrent mass at the same site; the soft tissue density and recurrent mass was basically the same as that of muscle, 3 patients had pericardial effusion. After contrast-enhanced scan, the soft tissue CT findings showed 5 cases of mild enhancement (solid part in cystic solid lesion and recurrent mass), and 1 case of moderate enhancement; the signal after MRI T1WI weighted fat sequence enhancement was basically the same as that of muscle (Table 2). The CT findings of localized mediastinal mesothelioma were basically the same as those localized abdominal mesothelioma described in the article of Liu, Y.et al [15] .
In conclusions, mediastinal mesothelioma is characterized by unclear etiology, no specific manifestations, high misdiagnosis rate and poor prognosis. Pericardial mesothelioma is remarkable. Early diagnosis is beneficial to the treatment of patients. The imaging findings of localized mesothelioma in the mediastinum have not been summarized in the previous literature. This paper presents a summary. Although the number of cases is small, it has certain imaging findings. The case suggests that when the pericardium has circumscribed thickening, the mediastinal tumor is closely connected to the pleura, with the manifestation of soft tissue density, mild-moderate enhancement after contrast-enhanced scan, and pericardial effusion; the possibility of mediastinal mesothelioma should be considered.

\section{Patient Perspective}

The patient shared ideas to decrease their burden of treatment and very satisfied with the treatment.

\section{Methods}

Formal approval was granted by the Zhuhai Hospital of Guangdong Hospital of traditional Chinese Medicine.

\section{Consent}

Written informed consent was obtained form 
the patient for the publication of this report and any accompanying images.

\section{Abbreviations}

MPM, malignant pleural mesothelioma; CT, Computed tomography; MRI, Magnetic resonance imaging; CTCA, computer tomography Coronary artery

\section{Availability of supporting data}

The datasets used or analysed during the current study are available from the corresponding author on reasonable request.

\section{Acknowledgements}

Grateful acknowledgement is made to Dr.Ben Bishop and Dr.Mary Macalalad. who gave me considerable help by means of suggestion.

\section{Authors'contributions}

Mengqiang xiao and Meng Zhang contributed equally to the present study. All authors read and approved the final manuscript.

\section{Ethics approval and consent to participate}

The requirement for informed consent was waived due to the retrospective nature of the study.

\section{Competing interests}

The authors declare that they have no competing interests.

\section{References}

1. Apicella G, Boulemden A, Citarella A, Sushma R, Szafranek A. Surgical treatment of a primary malignant pericardial mesothelioma: case report. Acta Chirurgica Belgica. 2020 03 18;:1-3. https://doi.org/10.1080/00015458.2020.1736869

2. Eren NT, Akar AR. primary pericardial mesothelioma. Current Treatment Options in Oncology. 2002 Oct;3(5):369-373. https://doi.org/10.1007/s11864-002-0002-7

3. Godar M, Liu J, Zhang P, Xia Y, Yuan Q. Primary Pericardial Mesothelioma: A Rare Entity. Case Reports in Oncological Medicine. 2013;2013:1-4. https://doi. org $/ 10.1155 / 2013 / 283601$

4. Akamoto S, Ono Y, Ota K, Suzaki N, Sasaki A, Matsuo $\mathrm{Y}$, Hayashi K. Localized malignant mesothelioma in the middle mediastinum: Report of a case. Surgery Today. 2008 07;38(7):635-638. https://doi.org/10.1007/s00595007-3679-1

5. ZHANG S, SONG P, ZHANG B. Giant malignant mesothelioma in the upper mediastinum: A case report. Oncology Letters. 201305 08;6(1):181-184. https://doi. org/10.3892/ol.2013.1333

6. Yang H, Testa JR, Carbone M. Mesothelioma Epidemiology, Carcinogenesis, and Pathogenesis. Current Treatment Options in Oncology. 2008 06;9(2-3):147-157. https://doi. org/10.1007/s11864-008-0067-z

7. Bendek M, Ferenc M, Freudenberg N. Post-irradiation pericardial malignant mesothelioma: an autopsy case and review of the literature. Cardiovascular Pathology. 2010 Nov;19(6):377-379. https://doi.org/10.1016/j. carpath.2009.08.003
8. Velissaris T, et al. Pericardial mesothelioma following mantle field radiotherapy. J Cardiovasc Surg (Torino). 2001;42(3):425-7.

9. Erdogan E, Demirkazık FB, Gulsun M, Arıyurek M, Emri S, Sak SD. Incidental localized (solitary) mediastinal malignant mesothelioma. The British Journal of Radiology. 2005 09;78(933):858-861. https://doi.org/10.1259/bjr/19513813

10. Griffin M, Farmer W, Garwood S, Lee D. Pericardial mesothelioma: An incidental intraoperative diagnosis. Journal of Cardiothoracic and Vascular Anesthesia. 1999 08;13(4):471-474. https://doi.org/10.1016/s10530770(99)90225-2

11. Sardar M, et al. Primary pericardial mesothelioma unique case and literature review. Tex Heart Inst J. 2012;39(2):2614.

12. Val-Bernal J, Figols J, Gómez-Román J. Incidental localized (solitary) epithelial mesothelioma of the pericardium. Cardiovascular Pathology. 2002 05;11(3):181-185. https:// doi.org/10.1016/s1054-8807(02)00097-2

13. Marchevsky AM, Khoor A, Walts AE, Nicholson AG, Zhang YZ, Roggli V, Carney J, Roden AC, Tazelaar HD, Larsen BT, LeStang N, Chirieac LR, Klebe S, Tsao M, De Perrot M, Pierre A, Hwang DM, Hung YP, Mino-Kenudson M, Travis W, Sauter J, Beasley MB, Galateau-Sallé F. Localized malignant mesothelioma, an unusual and poorly characterized neoplasm of serosal origin: best current evidence from the literature and the International Mesothelioma Panel. Modern Pathology. 201909 04;33(2):281-296. https://doi. org/10.1038/s41379-019-0352-3

14. Cao S, Jin S, Cao J, Shen J, Zhang H, Meng Q, Pan B, Yu Y. Malignant pericardial mesothelioma. Herz. 201701 27;43(1):61-68. https://doi.org/10.1007/s00059-016-4522-5

15. Liu Y, Wu J, Zhao Y, Zhang P, Hua Z, Dong W, Lin T, Liu A. Localized biphasic malignant mesothelioma presenting as a giant pelvic wall mass: a rare case report and literature review. BMC Medical Imaging. 202005 06;20(1). https:// doi.org/10.1186/s12880-020-00443-w

16. Sane, A.C, V.L. Roggli. Curative resection of a welldifferentiated papillary mesothelioma of the pericardium. Arch Pathol Lab Med. 1995;119(3):266-7.

17. Bierhoff, E, U. Pfeifer. Malignant mesothelioma arising from a benign mediastinal mesothelial cyst. Gen Diagn Pathol. 1996;142(1):59-62.

18. SHIMAZAKI H, AIDA S, IIZUKA Y, YOSHIZU H, TAMAJ S. Vacuolated cell mesothelioma of the pericardium resembling liposarcoma: A case report. Human Pathology. 2000 06;31(6):767-770. https://doi.org/10.1053/ hupa.2000.7630

19. Fujita K, Hata M, Sezai A, Minami K. Three-year survival after surgery for primary malignant pericardial mesothelioma: report of a case. Surgery Today. 201302 14;44(5):948-951. https://doi.org/10.1007/s00595-013$0511-\mathrm{y}$

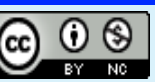

This work is licensed under a Creative Commons AttributionNon Commercial 4.0 International License. 